TRANSACTIONS OF THE

AMERICAN MATHEMATICAL SOCIETY

Volume 191, 1974

\title{
COMPLEX APPROXIMATION FOR VECTOR-VALUED FUNCTIONS WITH AN APPLICATION TO BOUNDARY BEHAVIOUR( ${ }^{1}$ )
}

\author{
BY
}

\author{
LEON BROWN, P. M. GAUTHIER AND W. SEIDEL
}

ABSTRACT. This paper deals with the qualitative theory of uniform approximation by holomorphic functions. The first theorem is an extension to vectorvalued mappings of $\mathrm{N}$. U. Arakélian's theorem on uniform holomorphic approximation on closed sets. Our second theorem is on asymptotic approximation and yields, as in the scalar case, applications to cluster sets.

Let $D$ be a proper domain of the Riemann sphere, $D^{*}$ its one-point compactification, $F$ a (relatively) closed subset of $D, X$ a locally convex (complex) topological vector space, $H(D, X)$ the holomorphic mappings from $D$ to $X, H_{F}(D, X)$ the uniform closure of restrictions to $F$ of mappings in $H(D, X)$, and $A(F, X)$ the continuous mappings from $F$ to $X$ holomorphic on $F^{0}$.

Mergelian's celebrated theorem [11] gives necessary and sufficient conditions in order that $H_{F}(D, X)=A(F, X)$, for the case where $D=X=\mathrm{C}$, and $F$ is compact. Recently, E. Briem, K. B. Laursen, and N. W. Pedersen [5] have extended Mergelian's theorem to the case where $D=C, F$ is compact, and $X$ is a locally convex space.

In a different direction Arakélian ([1], [2], and [3]) has generalized Mergelian's theorem to the case where $D$ is an arbitrary proper domain of the Riemann sphere, $F$ a (relatively) closed subset of $D$, and $X=\mathrm{C}$. Theorem 1 of the present paper extends Arakélian's theorem to the case where $X$ is a Fréchet space. We remark that the necessity of Arakélian's conditions holds for an arbitrary locally convex space.

The closed set $F \subset D$ is said to be a set of tangential, asymptotic, or Carleman approximation provided that for each $f \in A(F, C)$ and each positive continuous function $\epsilon$ on $F$, there is a function $g \in H(D, C)$ such that $|f(z)-g(z)|<\epsilon(z)$, $z \in F$. Arakélian [3] has given necessary and sufficient conditions for

Received by the editors June 11, 1973 and, in revised form, July 23, 1973.

AMS (MOS) subject classifications (1970). Primary 30A82, 30A96; Secondary 30 A72.

(1) Supported by NRC of.Canada, Grant A-5597, a grant from the Québec Government and the National Science Foundation, Grant \#20150. This work was done while Leon Brown was visiting the University of Michigan and P. M. Gauthier was visiting the University of Maryland. 
tangential approximation in case $F^{0}=\varnothing$. For general closed $F$, the problem has been completely solved by Nersesian [12]. Our Theorem 2 gives a vector-valued theorem on asymptotic approximation for the case where $F^{0}=\varnothing$. As in the scalar case (see [8]), Theorem 2 yields powerful corollaries on boundary behaviour of holomorphic functions. In the scalar case, asymptotic approximation has also found deep applications to value distribution theory [3].

The proof for Banach spaces is a modification of the proof in the scalar case and requires the use of the Hahn-Banach theorem at several points. We include it it in the Appendix as Theorem 3, partially for expository reasons, as the proof for the scalar case is not easily accessible and many details are left out. Our proof of Theorem 3 is modelled on Arakélian's proof for the case where $D=X=C$ as presented by W. Fuchs [7] and Arakélian's proof where $D$ is a general domain and $X=C$ [2].

We use the following notations. $S^{2}$ is the Riemann sphere; $\rho$ the chordal metric on $S^{2}$. For $E \subset S^{2}, \delta>0, N_{\rho}(E, \delta)=\left\{z \in S^{2}: \rho(E, z)<\delta\right\}$, and $\bar{E}$ denotes the $S^{2}$-closure of $E, \partial E$ the $S^{2}$-boundary. For $z \in \mathbf{C}, \delta>0, B(z, \delta)=\{\zeta \in \mathbf{C}$ : $|z-\zeta|<\delta\}$. Let $D$ be a proper domain in $S^{2}$. A boundary curve $\alpha$ in $D$ is a continuous curve $a:[0,1) \rightarrow D$ such that $a(t) \rightarrow \partial D$, as $t \rightarrow 1$. If $E$ and $X$ are topological spaces, $C(E, X)$ denotes the continuous mappings from $E$ to $X$; we write $C(E)=C(E, \mathrm{C})$ and $A(E)=A(E, \mathrm{C})$ etc. For $X$ a Fréchet space, $\left\{p_{n}\right\}$ will denote an increasing sequence of seminorms which generate the topology of $X$.

Theorem. 1. Let $X$ be a Fréchet space, $D$ a proper domain of the Riemann sphere, and $F$ a (relatively) closed subset of $D$. Then

$$
H_{F}(D, X)=A(F, X)
$$

if and only if $D^{*} \backslash F$ is connected and locally connected.

Proof. For the case where $X=C$, the necessity was shown by Arakélian [2] (see also [9]). Now suppose $D^{*} \backslash F$ is not locally connected and connected. Then there is a function $f \in A(F, \mathbf{C})$ and an $\epsilon>0$ such that

$$
|f(z)-g(z)|<\epsilon, \quad z \in F
$$

is satisfied by no function $g \in H(D, \mathrm{C})$. Fix $x \in X, x \neq 0$, and choose a seminorm $p$ such that $p(x)=1$. Then by the Hahn-Banach theorem, there is an $x^{*} \in X^{*}$ for which $x^{*}(x)=1$, and $\left|x^{*}(y)\right| \leq p(y)$, for all $y \in X$. Suppose, to obtain a contradiction, that (1) holds. Then there is a holomorphic mapping $G \in H(D, X)$ for which

$$
p(f(z) x-G(z))<\epsilon, z \in F \text {. }
$$

Thus

$$
\left|x^{*}(f(z) x-G(z))\right|<\epsilon
$$


that is, $\left|f(z)-x^{*}(G(z))\right|<\epsilon$, which contradicts (2) and proves the necessity.

To prove sufficiency it is enough to show that $A(F, X) \subset H_{F}(D, X)$ if $D^{*} \backslash F$ is connected and locally connected. Let $f \in A(F, X)$. We shall construct a Banach space $Y \subset X$, with a stronger topology such that $f$ is also in $A(F, Y)$. Since the theorem is true for Banach spaces (Theorem 3), $f \in H_{F}(D, Y)$. However, since the topology on $Y$ is stronger than the topology on $X, H_{F}(D, Y) \subset H_{F}(D, X)$, which will establish (1).

Let $\left\{p_{n}\right\}$ be a sequence of increasing seminorms which defines the topology for $X$. If $\left\{\omega_{n}\right\}$ is a sequence of positive numbers, then one easily shows that

$$
\left\{x \in X: \sup _{n} \omega_{n} p_{n}(x)<\infty\right\}=Y \subset X
$$

is a Banach space with a stronger topology than that of $X$. Let $\left\{D_{n}\right\}$ be an exhaustion of $D$ by domains: $\bar{D}_{n} \subset D_{n+1}, n=1,2, \cdots$, and $D=\bigcup_{n=1}^{\infty} D_{n}$. Set $K_{n}=F \cap D_{n}$, and let $K_{n}^{\prime}$ be an exhaustion of $F^{0}$ by compact sets such that $K_{n}^{\prime} \subset\left(K_{n+1}^{\prime}\right)^{0}, n=1,2, \cdots$, and $F^{0}=\bigcup_{n=1}^{\infty}\left(K_{n}^{\prime}\right)$. We set

$$
\omega_{n}^{\prime}=\frac{1}{1+\sup _{K_{n}} p_{n}(f(z))}, \quad \omega_{n}^{\prime \prime}=\frac{1}{1+\sup _{K_{n}^{\prime}} p_{n}\left(f^{\prime}(z)\right)} .
$$

Lemma 1. For eacb $j$,

$$
\sup _{z \in K_{j}} \sup _{n} \omega_{n}^{\prime} p_{n}(f(z))=M_{j}<\infty
$$

and

$$
\sup _{z \in K_{j}^{\prime}} \sup _{n} \omega_{n}^{\prime \prime} p_{n}\left(f^{\prime}(z)\right)=M_{j}^{\prime}<\infty
$$

Proof. If $z \in K_{j}$ and $n \geq j$, then $\omega_{n}^{\prime} p_{n}(f(z)) \leq 1$. While if $n<j, p_{n} \circ f$ is continuous and thus bounded on $K_{j}$. This proves (3), and (4) is proved similarly.

Now set

$$
\omega_{n}=\frac{\min \left(\omega_{n}^{\prime}, \omega_{n}^{\prime \prime}\right)}{n}
$$

and let $Y$ be the Banach space associated with the sequence $\left\{\omega_{n}\right\}$.

Lemma 2. With respect to the strong topology on $Y$,

(i) $f: F \rightarrow Y$ is continuous, and

(ii) $f: F^{0} \rightarrow Y$ is bolomorpbic.

Proof. Lemma 1 implies that $f(F) \subset Y$ and $f^{\prime}(F) \subset Y$, where $f^{\prime}$ is the derivative with respect to the topology of $X$.

To show (i) fix $z \in F$ and choose $j$ so that $z \in K_{j}$. Then, for $\zeta \in K_{j+1}$, 


$$
\begin{aligned}
\| f(z)- & f(\zeta) \|=\sup _{n} \omega_{n} p_{n}(f(z)-f(\zeta)) \\
& \leq \sup _{n \geq N} \omega_{n} p_{n}(f(z)-f(\zeta))+\sup _{n<N} \omega_{n} p_{n}(f(z)-f(\zeta)) \\
& \leq N^{-1} \sup _{n \geq N} \omega_{n}^{\prime} p_{n}(f(z)-f(\zeta))+\sup _{n<N} \omega_{n} p_{n}(f(z)-f(\zeta)) \\
& \leq M_{j+1} / N+\sup _{n<N} \omega_{n} p_{n}(f(z)-f(\zeta)) .
\end{aligned}
$$

Given $\epsilon>0$, choose $N>2 M_{j+1} / \epsilon$, and choose $\delta>0$ so that

$$
\zeta \in B(z, \delta) \cap F \Rightarrow \sup _{n<N} \omega_{n} p_{n}(f(z)-f(\zeta))<\epsilon / 2 .
$$

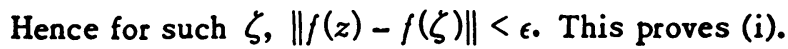

To show (ii), fix $z \in F^{0}$, and choose $j$ and $k$ such that $z \in K_{j} \cap K_{k}^{\prime}$. Now choose $\delta>0$ so that $B(z, \delta) \subset K_{j+1} \cap K_{k+1}^{\prime}$ and set $\Gamma=\{\zeta:|\zeta-z|=\delta\}$. If $|b|<\delta / 2$, then, for every $n$,

$$
\begin{aligned}
\omega_{n}^{\prime} p_{n}\left(\frac{f(z+b)-f(z)}{b}\right) & =\omega_{n}^{\prime} p_{n}\left(\frac{1}{b 2 \pi i} \int_{\Gamma} \frac{f(\zeta)}{\zeta-(z+b)}-\frac{f(\zeta)}{\zeta-z} d \zeta\right) \\
& \leq \frac{1}{2 \pi} \int_{\Gamma} \frac{\omega_{n}^{\prime} p_{n}(f(\zeta))|d \zeta|}{|[\zeta-(z+b)][\zeta-z]|} \\
& \leq M_{j+1} \frac{1}{2 \pi} \int_{\Gamma} \frac{|d \zeta|}{|[\zeta-(z+b)][\zeta-z]|} \leq \frac{2}{\delta} M_{j+1^{*}}
\end{aligned}
$$

Consequently

$$
\sup _{|b|<\delta / 2} \sup _{n} \omega_{n}^{\prime} p_{n}\left(\frac{f(z+b)-f(z)}{b}\right) \leq \frac{2}{\delta} M_{j+1} .
$$

Let $|b|<\delta / 2$ and $f^{\prime}$ be the derivative of $f$ with respect to the topology of $X$. Then

$$
\begin{aligned}
\left\|\frac{f(z+b)-f(z)}{b}-f^{\prime}(z)\right\| & =\sup _{n} \omega_{n} p_{n}\left(\frac{f(z+b)-f(z)}{b}-f^{\prime}(z)\right) \\
& \leq \sup _{n \geq N} \omega_{n} p_{n}()+\sup _{n<N} \omega_{n} p_{n}() .
\end{aligned}
$$

As in the proof of (i), it is sufficient to show that, for given $\epsilon$,

$$
\sup _{n \geq N} \omega_{n} p_{n}\left(\frac{f(z+b)-f(z)}{b}-f^{\prime}(z)\right)<\frac{\epsilon}{2},
$$

provided $N$ is sufficiently large. Now (7) is less than or equal to 


$$
\begin{aligned}
\sup _{n \geq N} \frac{\omega_{n}}{n} & p_{n}\left(\frac{f(z+b)-f(z)}{b}\right)+\sup _{n>N} \frac{\omega_{n}}{n} p_{n}\left(f^{\prime}(z)\right) \\
& \leq \frac{1}{N} \sup _{n \geq N} \omega_{n}^{\prime} p_{n}\left(\frac{f(z+b)-f(z)}{b}\right)+\frac{1}{N} \sup _{n \geq N} \omega_{n}^{\prime \prime} p_{n}\left(f^{\prime}(z)\right) \\
& \leq \frac{1}{N} \frac{2}{\delta} M_{j+1}+\frac{1}{N} M_{k}^{\prime} .
\end{aligned}
$$

The last inequality follows from (6) and (4). This establishes (7), which completes the proof of Lemma 2 and of the theorem.

Having considered the possibility of uniform approximation, we now turn to the possibility of asymptotic approximation.

Theorem 2. Let $X$ be a Fréchet space, $D$ a proper domain of the Riemann spbere, and $F$ a (relatively) closed subset of $D$ with empty interior. Then, for each mapping $f \in C(F, X)$ and positive function $\in \in C\left(D, \mathbf{R}^{+}\right)$, there is a bolomorphic mapping $g \in H(D, X)$ such that for each continuous seminorm $p$,

$$
p(f(z)-g(z))=o(\epsilon(z)),
$$

as $z \rightarrow \partial D, z \in F$, if and only if $D^{*} \backslash F$ is connected and locally connected.

Proof. In case $X=\mathrm{C}$, this theorem is essentially a corollary of Theorem 1 . However in the general case, it seems that a detailed proof is required.

We remark that it is sufficient to show that for $n=1,2, \ldots$

$$
p_{n}(f(z)-g(z))=O(\epsilon(z)),
$$

as $z \rightarrow \partial D, z \in F$. This is so, because for each $p$ there is an $n$ and a constant $A$ such that $p<A p_{n}$, and by choosing an appropriate $\epsilon(z)$ we can replace o by $O$.

We now prove (9). Let $D, F, f$, and $\epsilon$ be as in the hypothesis of the theorem. If $F$ is compact, then the theorem is vacuously true.

Suppose $F$ is not compact. Then we may assume $\lim \epsilon(z)$ is zero as $z$ approaches the boundary of $D$.

If $K$ is a compact set in $D$ we may choose a compact set $K^{1} \subset D$ and containing $K$ such that $\left(D^{*} \backslash F\right) \backslash K^{1}$ is connected. Also set

$$
K^{2}=\left\{z \in F: \epsilon(z) \geq \epsilon(\zeta) \text {, for some } \zeta \in K^{1}\right\} \cup K^{1} .
$$

Now let $\left\{K_{n}\right\}$ be a normal exhaustion of $D$ by compact sets inductively constructed so that $K_{0} \cap F \neq \varnothing$ and $K_{n-1}^{2} \subset K_{n}^{0}, n=1,2, \ldots$. We may choose the $K_{n}$ in such a manner so that the $\epsilon_{n}$ strictly decrease to zero, where $\epsilon_{n}=$ $\min \left\{\epsilon(z): z \in K_{n}^{2}\right\}$. Set $F_{0}=F \cap K_{0}^{2}$ and, for $n=1,2, \cdots$, set

$$
F_{n}=K_{n-1}^{2} \cup\left(F \cap K_{n}^{2}\right)=K_{n-1}^{2} \cup\left\{z \in F: \epsilon(z) \geq \epsilon_{n}\right\} \text {. }
$$

It is not difficult to verify that $D^{*} \backslash F_{n}$ is connected. Thus $F_{n}$ is a "Mergelian set." 
Set $f_{0}=f$, for $z \in F_{0}$. Then by Mergelian's theorem [ 5 ], there is a mapping $g_{0}$ holomorphic on all of $D$ such that $p_{1}\left(g_{0}(z)-f_{0}(z)\right)<\epsilon_{1} / 4, z \in F_{0}$. Now set $K_{-1}=\varnothing, \epsilon_{-1}=+\infty, g_{-1}=g_{0}$ and suppose mappings $g_{j}, j=0,1, \cdots, n-1$, holomorphic on all of $D$, have been defined satisfying

$$
\begin{array}{cl}
p_{j}\left(f(z)-g_{j}(z)\right) \leq \epsilon_{j} / 2, & \text { if } \epsilon_{j} \leq \epsilon(z) \leq \epsilon_{j-1}, \\
p_{j+1}\left(f(z)-g_{j}(z)\right) \leq \epsilon_{j+1} / 4, & \text { if } \epsilon(z)=\epsilon_{j},
\end{array}
$$

and

$$
p_{j}\left(g_{j}(z)-g_{j-1}(z)\right)<\epsilon_{j} / 2^{j+1}, \quad z \in K_{j-1}^{2} .
$$

We wish to define $g_{n}$, but first we must define $f_{n}$. Set $f_{n}=g_{n-1}$ on $K_{n-1}^{2}$. The function $f_{n}$ is defined on the closed set $K_{n-1}^{2}$ and we shall define it on all of $F_{n}$. Set $H_{n}=f_{n}-f$. Then $H_{n}$ is defined on a closed set which does not meet the set

$$
\left\{z \in F: \epsilon(z)=\epsilon_{n}\right\} \text {. }
$$

Set $H_{n}=0$ on the set (13). $H_{n}$ is continuously defined on a closed subset of the normal space

$$
\left\{z \in F: \epsilon_{n-1} \geq \epsilon(z) \geq \epsilon_{n}\right\}
$$

and is $p_{n}$-bounded by $\epsilon_{n} / 4$. By Dugundii's extension of Tietze's theorem [6], we extend $H_{n}$ continuously to all of (14) so that the extension retains the same bound. Now $f_{n}=f+H_{n}$ is continuously defined on all of $F_{n}$ and is holomorphic on the interior. By Mergelian's theorem, there is a function $g_{n}$ holomorphic on all of $D$ such that $p_{n+1}\left(g_{n}(z)-f_{n}(z)\right)<\epsilon_{n+1} / 2^{n+1}, z \in F_{n}$. From the way in which $f_{n}$ was defined, it follows that $g_{n}$ satisfies (10), (11), and (12).

Thus we define a sequence $\left\{g_{n}\right\}$ satisfying (10), (11), and (12). From (12) it is clear that $\left\{g_{n}\right\}$ converges to a mapping $g$ holomorphic on all of $D$. Now fix $p_{n}$ and let $z$ be any point of $F$ so close to the boundary of $D$ that $\epsilon(z) \leq \epsilon_{n-1}$. Choose $N$ so that $\epsilon_{n}(z) \leq \epsilon(z) \leq \epsilon_{N-1}$. Then for any $m>N$,

$$
p_{n}(f(z)-g(z)) \leq p_{n}\left(f(z)-g_{N}(z)\right)+\sum_{j=N+1}^{m} p_{j}\left(g_{j}(z)-g_{j-1}(z)\right)+p_{m}\left(g_{m}(z)-g(z)\right) \text {. }
$$

By choosing $m$ sufficiently large, the last term can be made as small as desired, and so from (10) and (12) it follows that $p_{n}(f(z)-g(z))<\epsilon{ }_{N} \leq \epsilon(z)$. Since this holds for any $z$ for which $\epsilon(z) \leq \epsilon_{n-1}$, the proof is complete.

As a consequence of the preceding proof we have

Corollary 1. Under the bypotheses of Theorem 2, for each seminorm p, there is a bolomorpbic mapping $g \in H(D, X)$ for which $p(f(z)-g(z))<\epsilon(z), z \in F$. 
As a further application of Theorem 2 we have the following result, first proved by Kaplan [10] for the case that $X=C$.

Corollary 2. Let $X$. be a Fréchet space, $D$ a proper domain of the Riemann sphere, $\left\{\alpha_{n}\right\}_{n=1}^{\infty}$ a family of disjoint boundary curves, and let $f \in C\left(\alpha_{n}, X\right), n=$ $1,2,3, \ldots$, and $\epsilon \in C\left(D, \mathbf{R}^{+}\right)$. Then there exists a bolomorphic mapping $g \in H(D, X)$ such that for each continuous seminorm $p$, and each $n=1,2, \cdots, p(f(z)-g(z))=$ $o(\epsilon(z))$, as $z \rightarrow \partial D$, along $a_{n}$.

Proof. The proof is the same as for the case $X=\mathrm{C}$ (see [8]).

A holomorphic mapping $g \in H(D, X)$ is said to have asymptotic value $x \in X$ along a boundary path $\alpha$ in $D$ provided $f(z) \rightarrow x$ as $z \rightarrow \partial D$ along $\alpha$.

Corollary 3. Let $X$ be a Fréchet space, $D$ a proper domain of the Riemann sphere, $a_{n}, n=1,2, \ldots$, a family of disjoint boundary curves, and $x_{n}, n=$ $1,2, \cdots$, a sequence of elements of $X$. Then there is a bolomorpbic mapping $g \in H(D, X)$ which bas asymptotic value $x_{n}$ along $a_{n}$, for $n=1,2, \ldots$.

Note that by an appropriate choice of $\epsilon(z)$, one also obtains a mapping which approaches prescribed values with a prescribed speed along prescribed boundary curves. For the case where $X=\mathrm{C}$, Corollary 3 was proved by Bagemihl and Seidel [4] and by Rudin [13].

We conclude this section with some open problems.

Conjecture 1. Theorem 1 fails if $X$ is allowed to be a general locally convex space. A counterexample is required.

Conjecture 2. Theorem 1 holds for $X$ locally convex, $K$ compact, and $D$ an open Riemann surface.

Conjecture 3. Theorem 2 holds for $X$ Fréchet and $D$ an open Riemann surface.

\section{APPENDIX}

Theorem 3. If $X$ is a Banach space then $H_{F}(D, X)=A(F, X)$ if and only if $D^{*} \backslash F$ is connected and locally connected.

Proof. The following proof is based upon Arakélian's proof for the case where $D$ and $X$ are the plane as presented by W. Fuchs [7] and Arakélian's proof where $D$ is a general domain and $X$ the plane [2].

Four lemmas are required. We begin with a slightly modified lemma of Rudin.

Lemma 1 (Rudin [14, p. 383]). Suppose $\sigma$ is a compact and connected sub. set contained in a disc of diameter $2 r, \Omega=S^{2} \backslash \sigma$ is connected, and the diameter of $\sigma$ is at least $.99 r$. Then there is a function $g \in H(\Omega)$ and a constant $b$, with the following property: If

$$
Q(\zeta, z)=g(z)+(\zeta-b) g^{2}(z),
$$


the inequalities

$$
\begin{gathered}
|Q(\zeta, z)|<100 / r \\
\left|Q(\zeta, z)-1 \prime^{\prime}(z-\zeta)\right|<1000 r^{2} /|z-\zeta|^{3},
\end{gathered}
$$

bold for all $z \in \Omega$ and for all $\zeta$ in the disc.

Lemma 2. Let $\sigma$ be a compact, connected subset of $D$ and $\gamma$ be a boundary curve originating at a point of $\sigma, E_{0}$ a closed subset of $D$ such that $(\gamma \cup \sigma) \cap$ $E_{0}=\varnothing$. If $Q(z)$ is a function bolomorpbic in $S^{2} \backslash \sigma, \eta>0$, then there exists a $\psi$ in $H(D)$ such that

$$
|\psi(z)-Q(z)|<\eta \quad \text { for } z \in E_{0} .
$$

Proof. The method of proof involves the standard technique of pole pushing. We include the proof for completeness.

Let $\gamma$ be parameterized by $z:[0,1) \rightarrow D$. By finite induction one chooses $0<t_{1}<t_{2}<\ldots<t_{n}<t_{n+1}<\ldots<1$ such that if $t \geq t_{n}$ then $\rho(z(t), \partial D)<1 / n$. Again by induction we choose domains $G_{n}$ such that

(i) $\bar{G}_{n} \cap E_{0}=\varnothing$ for all $n$,

(ii) $G_{1} \supset \sigma \cup z\left(\left[0, t_{1}\right)\right)$,

(iii) $G_{n} \supset z\left(\left[t_{n-1}, t_{n}\right]\right), n>1$,

(iv) $G_{n} \subset N_{\rho}(\partial D, 1 /(n-2)), n>2$.

Using Runge's theorem we construct inductively a sequence of rational functions $\psi_{n}$ with the following properties:

(i) $\psi_{n}$ has a unique pole at $z\left(t_{n}\right)$,

(ii) $\left|Q(z)-\psi_{1}(z)\right|<\eta / 2$ for $z \in S^{2} \backslash G_{1}$,

(iii) $\left|\psi_{n-1}(z)-\psi_{n}(z)\right|<\eta / 2^{n}$ for $z \in S^{2} \backslash G_{n}$ and $n>1$.

On compact subsets of $D, \psi_{n}$ converges uniformly to a function $\psi$ in $H(D)$. If $z \in E_{0}$, then

$$
|Q(z)-\psi(z)| \leq\left|Q(z)-\psi_{1}(z)\right|+\sum_{n=2}^{\infty}\left|\psi_{n-1}(z)-\psi_{n}(z)\right|<\eta .
$$

This completes the proof of the lemma.

Lemma 3. Let $D$ be a domain of the finite plane. Then there exists a function $\rho \in C^{\infty}(D)$ such that $0<\rho(z)<\rho(z, \partial D),|\bar{\partial} \rho|<1$ for $z \in D$.

Proof. See Arakélian [2, Lemma 4].

If $\phi$ is a function from $D$ into $X$ which has continuous partial derivatives we say that $\phi \in C^{1}(\dot{D}, X)$. 
Lemma 4. Let $D$ be a domain of the finite plane and $f$ a continuous func. tion from $D$ to $X$. For $\epsilon>0$, there exist $\delta \in C^{1}(C)$ and $\phi \in C^{1}(D, X)$ such that $\left.\delta\right|_{C \backslash D}=0$, and for $z \in D$,

$$
\begin{gathered}
0<\delta(z)<1 / 2 \rho(z, \partial D), \quad|\bar{\partial} \delta|<\min \left\{\epsilon, \delta^{1 / 2}(z)\right\}, \\
\|\phi(z)-f(z)\|<\epsilon, \quad\|\bar{\partial} \phi(z)\|<\epsilon / \delta(z) .
\end{gathered}
$$

Furthermore, if for some point $z \in D$, the function $f$ is bolomorpbic in the disc $|\zeta-z|<\delta(z)$, then $\phi(z)=f(z)$.

Proof. Taking $\epsilon<1$, we shall construct the function $\delta(z)$. For the compact set $K_{\lambda} \subset \mathbf{C}, K=\{z \in D: \rho(z, \partial D) \geq \lambda\}$, the condition $z \in K_{\lambda}$ and $|\zeta-z| \leq \lambda / 2$ implies that $\zeta \in K_{\lambda / 2}$. Thus for $\delta$ a constant, $\delta \leq \lambda / 2$, we may set

$$
\omega(\lambda, \delta)=\max _{z \in K_{\lambda},|\zeta-z| \leq \delta}\|f(\zeta)-f(z)\| \text {. }
$$

Note that $\omega(\cdot, \delta)$ is nonincreasing, $\omega(\lambda, \cdot)$ is nondecreasing and $\omega(\lambda, 0)=0$.

Thus we may define $\delta_{1}(\lambda)$ as the unique value of $\delta$ for which $\omega(\lambda, \delta)+\delta=\epsilon \lambda / 2$. Note that $\delta_{1}(\lambda)$ is strictly increasing. Now define $\delta_{2}(\lambda)$ by

$$
\delta_{2}^{1 / 2}(\lambda)=1 / 2 \int_{0}^{\lambda} \delta_{1}^{1 / 2}(t) d t, \quad 0<\lambda<1
$$

Evidently, we have

$$
\delta_{2}(\lambda)<\delta_{1}(\lambda) \leq \epsilon \lambda / 2<\lambda / 2
$$

Also, a direct calculation gives

$$
\delta_{2}^{\prime}(\lambda)=\delta_{2}^{1 / 2}(\lambda) \delta_{1}^{1 / 2}(\lambda)<\min \left\{\epsilon / 2, \delta_{2}^{1 / 2}(\lambda)\right\} .
$$

For $z \in D$, we set $\delta(z)=\delta_{2}(\rho(z))$, where $\rho$ is the function in Lemma 3. (19) follows since

$$
0<\delta(z)=\delta_{2}(\rho(z))<\rho(z) / 2<\rho(z, \partial D) / 2
$$

and

$$
|\bar{\partial} \delta(z)|=\left|\delta_{2}^{\prime}(\rho(z)) \| \bar{\partial} \rho(z)\right| \text {. }
$$

For $z \in C \backslash D$, set $\delta(z)=0$. We must verify that $\delta \in C^{1}(C)$. This follows from (21) and the fact that $\delta_{2}^{\prime}$ is continuous and $\rho \in C^{1}(D)$.

The mapping $\phi$ is defined by the vector-valued integral

$$
\phi(z)=\left\{\pi \delta^{2}(z)\right\}^{-1} \iint_{|\zeta-z|<\delta(z)} f(\zeta) d s_{\zeta},
$$

where $d s_{\zeta}$ is Lebesgue planar measure. It is clear from (22) that if $f$ is holomorphic in the disc $|\zeta-z|<\delta(z)$, then $x^{*}(\phi(z))=x^{*}(f(z))$, for each $x^{*} \in X^{*}$, 
which implies by the Hahn-Banach theorem, that $\phi(z)=f(z)$.

Furthermore

$$
\begin{aligned}
\|\phi(z)-f(z)\| & \leq \max _{|\zeta-z| \leq \delta(z)}\|f(\zeta)-f(z)\| \leq \omega(\rho(z), \delta(z)) \\
& =\omega\left(\rho(z), \delta_{2}(\rho(z))\right) \leq \omega\left(\rho(z), \delta_{1}(\rho(z))\right)<\epsilon \rho(z) / 2<\epsilon / 2<\epsilon .
\end{aligned}
$$

To complete the proof, there remains only to verify the second inequality of (20).

$$
\bar{\partial} \phi(z)=-\frac{2 \bar{\partial} \delta(z)}{\pi \delta^{3}(z)} \iint_{|\zeta-z| \leq \delta(z)} f(\zeta) d s_{\zeta}+\frac{1}{\pi \delta^{2}(z)} \bar{\partial} \iint_{|\zeta-z| \leq \delta(z)} f(\zeta) d s_{\zeta}
$$

Let $\psi(z, \delta)=\iint|\zeta-z|<\delta f(\zeta) d s_{\zeta}$. Then

Since $\bar{\partial} z=0$, we have

$$
\bar{\partial} \psi(z, \delta)=\left[\frac{\partial \psi}{\partial \delta}(z, \delta) \bar{\partial} \delta(z)+\frac{\partial \psi}{\partial z}(z, \delta) \bar{\partial} z\right]_{\delta=\delta(z)^{\circ}}
$$

$$
\begin{aligned}
\bar{\partial} \phi(z) & =-\frac{2 \bar{\partial} \delta(z)}{\delta(z)} \phi(z)+\frac{1}{\pi \delta^{2}(z)}\left(\frac{\partial}{\partial \delta} \iint_{|\zeta-z| \leq \delta} f(\zeta) d s_{\zeta}\right) \bar{\partial} \delta(z) \\
& =-\frac{2}{\delta(z)} \bar{\partial} \delta(z) \cdot \phi(z)+\frac{1}{\pi \delta^{2}(z)}\left[\frac{\partial}{\partial \delta} \int_{0}^{\delta} \int_{0}^{2 \pi} f\left(z+u e^{i \theta}\right) u d \theta d u\right] \bar{\partial} \delta(z) \\
& =\frac{2 \bar{\partial} \delta(z)}{\delta(z)}\left[-\phi(z)+\frac{1}{2 \pi} \int_{0}^{2 \pi} f\left(z+\delta(z) e^{i \theta}\right) d \theta\right] \\
& =\frac{2 \bar{\partial} \delta(z)}{\delta(z)}\left[f(z)-\phi(z)+\frac{1}{2 \pi} \int_{0}^{2 \pi}\left\{f\left(z+\delta(z) e^{i \theta}\right)-f(z)\right\} d \theta\right] .
\end{aligned}
$$

Thus,

$$
\|\bar{\partial} \phi(z)\| \leq \frac{2 \epsilon}{\delta(z)}\left[\epsilon+\frac{1}{2 \pi} \int_{0}^{2 \pi} \omega(\rho(z), \delta(z)) d \theta\right]<\frac{3 \epsilon^{2}}{\delta(z)},
$$

and the proof of Lemma 4 is complete.

Having established the required lemmas, we now proceed with the proof of the theorem. Let $D^{*} \backslash F$ be connected and locally connected. If $f \in A(F, X)$, then we shall prove that $f \in H_{F}(D, X)$. Since $X$ is an absolute retract [6], we may assume that $f \in C(D, X)$. We may also assume $\infty \notin D$. Given $\epsilon>0$, let $\phi$ and $\delta$ be the functions from Lemma 4 , and $T$ the support of $\partial \phi$. Also,let $\left\{D_{n}\right\}$ be a normal exhaustion of $D$. That is, for each $n, D_{n}$ is a domain bounded by finitely many disjoint smooth Jordan curves, $\bar{D}_{n} \subset D_{n+1}$, and $D=\bigcup_{n=1}^{\infty} D_{n}$. Using the Hahn-Banach theorem, one easily obtains the following generalization of Pompeiu's formula [7, p. 14]

$$
\phi(z)=\frac{1}{2 \pi i} \int_{\partial D_{n}} \frac{\phi(\zeta)}{\zeta-z} d \zeta-\frac{1}{\pi} \iint_{D_{n}} \frac{\bar{\phi}(\zeta)}{\zeta-z} d s_{\zeta}
$$

for $z \in D_{n}$. 
Let $\zeta_{0}$ be such that $d\left(\zeta_{0}, D \backslash F\right) \leq \delta\left(\zeta_{0}\right)$. Therefore there is a point $\zeta^{\prime} \in(D \backslash F) \cap B\left(\zeta_{0}, 1.001 \delta\left(\zeta_{0}\right)\right)$. Consequently, since $D^{*} \backslash F$ is connected and locally connected $\zeta^{\prime}$ can be joined to the boundary of $D$ by a curve $\gamma_{0} \subset D \backslash F$, such that for each $m$ there is an $n>m$ such that

$$
\gamma_{0} \cap D_{m}=\varnothing \quad \text { if } \zeta_{0} \in D \backslash D_{n} \text {. }
$$

Moreover, one can find an arc $\sigma_{0}$ of $\gamma_{0}$ contained in $B\left(\zeta_{0}, 2 \delta\left(\zeta_{0}\right)\right)$ and of diameter $0.99 \delta\left(\zeta_{0}\right)$. An application of Lemma 1 establishes the existence of a function $Q_{0}(\zeta, z)$ linear in $\zeta, Q_{0}(\zeta, \cdot) \in H\left(S^{2} \backslash \sigma_{0}\right)$,

$$
\left|Q_{0}(\zeta, z)\right|<\frac{100}{\delta\left(\zeta_{0}\right)}, \quad\left|Q_{0}(\zeta, z)-\frac{1}{\zeta-z}\right|<\frac{1000 \delta^{2}\left(\zeta_{0}\right)}{|\zeta-z|^{3}},
$$

where $\zeta \in B\left(\zeta_{0}, 2 \delta\left(\zeta_{0}\right)\right)$ and $z \in S^{2} \backslash \sigma_{0}$. By restricting $\zeta$ to a sufficiently small disc $B_{\zeta_{0}}$ with center at $\zeta_{0}$ we arrive at the following inequalities:

$$
\begin{gathered}
\left|Q_{0}(\zeta, z)\right|<200 / \delta(\zeta), \\
\left|Q_{0}(\zeta, z)-1 /(\zeta-z)\right|<2000 \delta^{2}(\zeta) /|\zeta-z|^{3},
\end{gathered}
$$

where $\zeta \in B_{\zeta_{0}}$ and $z \in S^{2} \backslash \sigma_{0}$.

The family $\left\{B_{\zeta}: \zeta \in T\right\}=\mathscr{B}$ is an open cover of $\bar{D}_{n} \cap T$ for all $n$. By finite induction and the Heine-Borel theorem we can extract a sequence $B_{n}$ with associated functions $Q_{n}(\zeta, z), n=1,2, \ldots$, such that $T \cap \bigcup_{1}^{n} B_{j}$ is strictly increasing and for a subsequence of indices $n_{k}, \bar{D}_{k} \cap T \subset \bigcup_{j=1}^{n_{k}} B_{j}$.

For $\zeta \in T$, we define $Q(\zeta, z)=Q_{j}(\zeta, z)$, where $j$ is the smallest index such that $\zeta \in B_{j}$. Let $G_{n}=T \cap\left(B_{n} \backslash \bigcup_{1}^{n-1} B_{j}\right)$. Then we have If $\zeta \in G_{n}, Q(\zeta, \cdot)$ is holomorphic on $\Omega_{\zeta}=S^{2} \backslash \sigma_{n} \supset F$, where $\sigma_{n}$ is an arc of a curve which joins a point near $\zeta$ to the boundary of $D$,

$$
Q(\cdot, z) \text { is linear on } G_{n} \text {. }
$$


exists by (27), and the fact that $T \cap D_{n}$ is contained in a finite union of the $G_{k}$ 's. We shall show that

$$
\|I\|<A \epsilon,
$$

where $A$ is a constant independent of $n$ and of $z \in F$. For $V_{z}=\{\zeta \in D:|\zeta-z|<$ $4 \delta(\zeta)\}$

$$
\begin{aligned}
\|I\| & \leq \frac{1}{\pi} \iint_{T \cap D_{n}} \frac{\epsilon}{\delta(\zeta)}\left|Q(\zeta, z)-\frac{1}{\zeta-z}\right| d s_{\zeta} \\
& =\iint_{T \cap D_{n} \cap V_{z}}+\iint_{T \cap D_{n} \backslash V_{z} \leq A \epsilon\left[I_{1}+I_{2}\right],}
\end{aligned}
$$

by (29) and (30), where

$$
I_{1}=\iint_{V_{z}} \frac{d s_{\zeta}}{\delta(\zeta)|\zeta-z|}, \quad I_{2}=\iint_{C \backslash V_{z}} \frac{\delta(\zeta)}{|\zeta-z|^{3}} d s_{\zeta}
$$

Because of (19) and the mean-value theorem,

$$
|\delta(\zeta)-\delta(z)| \leq 2 \epsilon|\zeta-z|, \quad \zeta, z \in D .
$$

Hence if $\epsilon<1 / 16$, we obtain for $\zeta \in V_{z}, \delta(z) / 2<\delta(\zeta)<2 \delta(z)$, which implies that $V_{z} \subset B(z, 8 \delta(z))$. Therefore

$$
I_{1}<\frac{2}{\delta(z)} \iint_{B(z, 8 \delta(z)} \frac{d s \zeta}{|\zeta-z|}=32 \pi
$$

Analogously $C \backslash V_{z} \subset C \backslash B(z, 2 \delta(z))$ and so

$$
I_{2}<2 \pi+\iint_{B(z, 1) \backslash B(z, 2 \delta(z))} \frac{\delta(\zeta)}{|\zeta-z|^{3}} d s_{\zeta}=2 \pi+\int_{0}^{2 \pi} I_{3}(\theta) d \theta .
$$

Furthermore, taking (19) into account, and the estimate $\delta(\zeta) \leq|\zeta-z|, \zeta \epsilon$ $C \backslash B(z, 2 \delta(z))$, and integrating by parts,

$$
\begin{aligned}
I_{3} & =-\int_{2 \delta(z)}^{1} \delta\left(z+\rho e^{i \theta}\right) d(1 / \rho) \\
& <\frac{\delta\left(z+2 \delta(z) e^{i \theta}\right)}{2 \delta(z)}+\int_{2 \delta(z)}^{1} \frac{\delta^{1 / 2}\left(z+\rho e^{i \theta}\right)}{\rho} d \rho<1+\int_{0}^{1} \frac{d \rho}{\sqrt{\rho}}=3 .
\end{aligned}
$$

This completes the proof of (31); which says that the function

satisfies

$$
b_{n}(z)=\frac{1}{2 \pi} \int_{\partial D_{n}} \frac{\phi(\zeta)}{\zeta-z} d \zeta-\frac{1}{\pi} \iint_{T \cap D_{n}} \overline{\partial \phi(\zeta) Q(\zeta, z) d s_{\zeta}}
$$

$$
\left|\phi(z)-b_{n}(z)\right|<A \epsilon, \quad z \in F \cap D_{n},
$$

where $A$ is a constant independent of $n$.

Recalling that for $\zeta \in G_{j}, Q(\zeta, z)=g_{j}(z)+\left(\zeta-b_{j}\right) g_{j}^{2}(z)$, where $g_{j}$ is holomorphic in $S^{2} \backslash \sigma_{j}$, we invoke Lemma 2 to obtain a function $\psi_{j}(z)$ holomorphic in $D$ such that 


$$
\left|g_{j}(z)-\psi_{j}(z)\right|<\eta_{j}, \quad z \in F \cup \bar{D}_{k(j)},
$$

where $k(j)$ is the largest index for which $\bar{D}_{k}$ does not meet the boundary curve $\gamma_{j}$ of which $\sigma_{j}$ is an arc, and $\eta_{j}$ is chosen so small that setting

$$
G(\zeta, z)=\psi_{j}(z)+\left(\zeta-b_{j}\right) \psi_{j}^{2}(z)
$$

$\zeta \in G_{j}, z \in D$, we have

$$
|G(\zeta, z)-Q(\zeta, z)|<\delta(\zeta) /\left(1+|\zeta|^{3}\right),
$$

for $\zeta \in G_{j}$ and $z \in F \cup \bar{D}_{k(j)}$. From (35), $G(., z)$ is integrable over $T \cap D_{n}$, and we have

$$
\begin{aligned}
\| \frac{1}{\pi} \iint_{T \cap D_{n}} \overline{\partial \phi}(\zeta)\{Q(\zeta, z)-G(\zeta, z)\} d s \zeta & \leq \frac{1}{\pi} \iint_{T \cap D_{n}} \frac{\epsilon}{\delta(\zeta)} \frac{\delta(\zeta)}{1+|\zeta|^{3}} d s_{\zeta} \\
& \leq \frac{\epsilon}{\pi} \iint_{C} \frac{d s_{\zeta}}{1+|\zeta|^{3}}=A \epsilon,
\end{aligned}
$$

for $z \in F$, where $A$ is a constant independent of $z$ and of $n$.

Thus we have shown that the function

$$
H_{n}(z)=\frac{1}{2 \pi i} \int_{\partial D_{n}} \frac{\phi(\zeta)}{\zeta-z} d \zeta-\frac{1}{\pi} \iint_{T \cap D_{n}} \bar{\partial} \phi(\zeta) G(\zeta, z) d s \zeta
$$

holomorphic in $D_{n}$, satisfies

$$
\left\|f(z)-H_{n}(z)\right\|<A \epsilon, \quad z \in F \cap D_{n},
$$

where $A$ is independent of $n$ and $z$.

To complete the proof we need only show that as $n \rightarrow \infty, H_{n}$ converges to a mapping $g$, holomorphic on all of $D$. Let $K$ be a compact set in $D$. Choose $n_{0}$ such that $K \subset D_{n_{0}}$. By (24) there is an $N_{1}$ such that $j \geq N_{1} \Rightarrow k(j) \geq n_{0}$. Now choose $N_{2}>N_{1}$ such that $T \backslash D_{N_{2}} \subset \bigcup_{j=N_{1}}^{\infty} G_{j}$. Suppose $N_{2}<m<n$. Then, for $z \in K$,

$H_{n}(z)-H_{m}(z)=\frac{1}{2 \pi i} \quad\left(\int_{\partial D_{n}}-\int_{\partial D_{m}}\right) \frac{\phi(\zeta)}{\zeta-z} d \zeta-\frac{1}{\pi} \iint_{T \cap\left(D_{n} \backslash D_{m}\right)} \bar{\partial} \phi(\zeta) G(\zeta, z) d s \zeta$.

The formula of Pompeiu then gives:

$$
\begin{aligned}
H_{n}(z)-H_{m}(z)= & \frac{1}{\pi} \iint_{T \cap\left(D_{n} \backslash D_{m}\right)} \bar{\partial}(\zeta)\left\{\frac{1}{\zeta-z}-G(\zeta, z)\right\} d s_{\zeta} \\
= & \frac{1}{\pi} \iint_{T \cap\left(D_{n} \backslash D_{m}\right)} \bar{\phi}(\zeta)\left\{\frac{1}{\zeta-z}-Q(\zeta, z)\right\} d s_{\zeta} \\
& +\frac{1}{\pi} \iint_{T \cap\left(D_{n} \backslash D_{m}\right)} \partial \phi(\zeta)\{Q(\zeta, z)-G(\zeta, z)\} d s_{\zeta} \\
= & I_{1}+I_{2} .
\end{aligned}
$$


The choice of $N_{2}$ yields the following estimate:

$$
\begin{aligned}
\left\|I_{1}\right\| & \leq \frac{1}{\pi} \iint_{D_{n} \backslash D_{m}} \frac{\epsilon}{\delta(\zeta)} \frac{2000 \delta^{2}(\zeta)}{|\zeta-z|^{3}} d s_{\zeta} \leq \frac{2000}{\pi} \iint_{D_{n} \backslash D_{m}} \frac{d s \zeta}{|\zeta-z|^{3}} ; \\
\left\|I_{2}\right\| & \leq \frac{1}{\pi} \iint_{D_{n} \backslash D_{m}} \frac{\epsilon}{\delta(\zeta)} \frac{\delta(\zeta)}{1+|\zeta|^{3}} d s_{\zeta}<\frac{1}{\pi} \iint_{D_{n} \backslash D_{m}} \frac{d s_{\zeta}}{1+|\zeta|^{3}} .
\end{aligned}
$$

These estimates show that $I_{1}$ and $I_{2}$ converge to zero, uniformly on $K$, as $n, m \rightarrow \infty$. Thus $H_{n}$ converges, uniformly on compact subsets of $D$, and the proof of Theorem 3 is complete.

\section{REFERENCES}

1. N. U. Arakélian, Uniform approximation on closed sets by entire functions, Izv. Akad. Nauk SSSR Ser. Mat. 28 (1964), 1187-1206. (Russian) MR 30 \#258.

2. - - Uniform and tangential approximation by analytic functions, Izv. Akad. Nauk Armjan. SSR Ser. Mat. 3 (1968), no. 4-5, 273-286. (Russian) MR 43 \#530.

3. - Approximation complexe et propriétés des fonctions analytiques, Actes, Congrès Intern. Math., 1970, Tome 2, 595-600.

4. F. Bagemihl and W. Seidel, Spiral and other asymptotic paths and paths of complete indetermination of analytic and meromorphic functions, Proc. Nat. Acad. Sci. U.S.A. 39 (1953), 1251-1258. MR 15, 515.

5. E. Briem, K. B. Laursen and N. W. Pedersen, Mergelian's theorem for vector-valued functions with an application to slice algebras, Studia Math. 35 (1970), 221-226. MR 43 \#3806.

6. J. Dugundji, An extension of Tietze's theorem, Pacific J. Math. 1 (1951), 353-367. MR 13, 373.

7. W. H. J. Fuchs, Théorie de l'approximation des fonctions d'une variable complexe, Séminaire de Mathématiques Supérieures, no. 26 (Été, 1967), Les Presses de l'Université de Montréal, Montréal, Que., 1968. MR 41 \#5630.

8. P. M. Gauthier and W. Seidel, Some applications of Arakélian's approximation theorems to the the ory of cluster sets, Izv. Akad. Nauk Armjan. SSR Ser. Mat. 6 (1971) 458-464.

9. P. M. Gauthier and W. Hengartner, Uniform approximation on closed sets by functions analytic on a Riemann surface, Proceedings, Conference on Approximation Theory, Poznan, 1972 (to appear).

10. W. Kaplan, Approximation by entire functions, Michigan Math. J. 3 (1955), 43-52. MR 17, 31 .

11. S. N. Mergelian, Uniform approximations of functions of a complex variable, Uspehi Mat. Nauk 7 (1952), no. 2 (48), 31-122; English transl., Amer. Math. Soc. Transl. (1) 3 (1962), 294-391. MR 14, 547.

12. A. A. Nersesjan, Carleman sets, Izv. Akad. Nauk Armjan. SSR Ser. Mat. 6 (1971), no. 6, 465-471. (Russian) MR $46 \# 367$. 
13. W. Rudin, Radial cluster sets of analytic functions, Bull. Amer. Math. Soc. 60 (1954), 545.

14. - Real and complex analysis, McGraw.Hill, New York, 1966. MR 35 \# 1420. DEPARTMENT OF MATHEMATICS, WAYNE STATE UNIVERSITY, DETROIT, MICHIGAN 48202

DÉPARTEMENT DE MATHÉMATIQUES, UNIVERSITÉ DE MONTRÉ, MONTRÉAL, QUÉBEC, CANADA 